\title{
The Young Investigator Retreat of the Canadian Critical Care Trials Group: mentorship and self-discovery
}

\author{
Ian M. Ball, MD, MSc $\cdot$ Lisa Burry, BScPharm, PharmD $\cdot$ Peter Dodek, MD, MHSc $\cdot$ Jamie S. Hutchison, MD \\ Jeanna Parsons Leigh, PhD • Victoria McCredie, MBChB, MRCP, PhD $\cdot$ Srinivas Murthy, MD, CM, MHSc • \\ Bram Rochwerg, MD, MSc - Deborah Cook, MD, MSc • for the Canadian Critical Care Trials Group and the \\ Canadian Critical Care Translational Biology Group
}

Received: 7 June 2017/Accepted: 9 November 2017/Published online: 17 November 2017

(C) Canadian Anesthesiologists' Society 2017

The Canadian Critical Care Trials Group (CCCTG) was created in 1989 to improve the care of critically ill patients through investigator-initiated research and to provide a national forum for continuing education about research methods. ${ }^{1}$ In 2004, a group of clinical and basic scientists interested in the basic mechanisms of critical illness formed the Canadian Critical Care Translational Biology Group (CCCTBG). The two groups have grown from the original 25 participants in 1989 to over 300 researchers today. The CCCTG and CCCTBG meet together and currently support over 40 research programs with more than 100 peer-reviewed publications to their credit. The

I. M. Ball, MD, MSc ( ()

Departments of Medicine, Epidemiology and Biostatistics,

Western University, London, ON, Canada

e-mail: Ian.Ball@lhsc.on.ca

L. Burry, BScPharm, PharmD

Department of Pharmacy and Medicine, Sinai Health System,

Toronto, ON, Canada

P. Dodek, MD, MHSc

Division of Critical Care Medicine and Centre for Health

Evaluation and Outcome Sciences, University of British

Columbia, Vancouver, BC, Canada

\section{J. S. Hutchison, MD}

Department of Critical Care and Neuroscience and Mental Health Research Program, Hospital for Sick Children, Toronto, ON, Canada

\section{J. S. Hutchison, MD}

Interdepartmental Division of Critical Care and Institute for Medical Science, University of Toronto, Toronto, ON, Canada most important ingredients for this success are the collegiality and mentorship of these groups.

A research mentor has many potential roles in developing the career of a young investigator, some of which include acting as advisor, advocate, coach, or supervisor, guiding the mentee with intent, commitment, and spirit. Given the need for sustainability and growth, the CCCTG and CCCTBG have an explicit mandate to mentor their junior and senior investigators and to foster their independent success. This mandate is addressed through several activities, including the "community mentoring" model of our thrice yearly meetings, explicit linkage between each young investigator who presents at these meetings and a CCCTG mentor, encouragement of junior members to participate in national committees, interdisciplinary young investigator grants, the Deborah J. Cook Mentorship Award to honour individuals who have made exceptional contributions in this domain, and the creation of the CCCTG Young Investigator Retreat. Dr.

\author{
J. P. Leigh, $\mathrm{PhD}$ \\ Department of Critical Care Medicine and the O'Brien Institute \\ for Public Health, University of Calgary, Calgary, AB, Canada \\ V. McCredie, MBChB, MRCP, PhD \\ Department of Medicine, University of Toronto, Toronto, ON, \\ Canada \\ S. Murthy, MD, CM, MHSc \\ Department of Pediatrics, University of British Columbia, \\ Vancouver, BC, Canada \\ B. Rochwerg, MD, MSc · D. Cook, MD, MSc \\ Departments of Medicine, Clinical Epidemiology \& \\ Biostatistics, McMaster University, Hamilton, ON, Canada
}


Deborah Cook and her partner Robert hosted the inaugural CCCTG Young Investigator Retreat. It was largely modelled after the Trout Lake Workshops founded by the late Dr. David Sackett, one of Dr. Cook's most influential mentors and a pioneer of evidence-based medicine. ${ }^{2-6}$

\section{Funding and participant selection}

In 2014, the CCCTG received a Canadian Institutes of Health Research (CIHR) Community Development Grant, some of which was allocated to support mentorship. Members of CCCTG in their first five years of a research position were invited to apply for the inaugural 2016 Young Investigator Awards. The four-member CCCTG Career Development \& Mentorship Committee reviewed and evaluated the applications (a proposed study summary and letter from the research supervisor/mentor) on three domains-i.e., potential impact of the retreat on the individual and their organization, clarity and feasibility of the proposed study summary, and potential impact of the proposed research on patient outcomes. By consensus, participants were selected from across Canada and from several professional disciplines: two adult intensivists, one neurointensivist, one pediatric intensivist, two doctoral pharmacists, and one doctoral sociologist. This multidisciplinary approach mirrors many effective investigator-led CCCTG research programs (a key aspect of the CIHR Community Development Grant). These seven participants varied with respect to their research foci and methods, which included clinical and health services research and international pandemic preparedness research. Three senior critical care investigators (two adult and one pediatric) served as mentors. These mentors were selected based on their excellent track records as accomplished clinical researchers and their known commitment to teaching and mentorship.

\section{Retreat format}

The retreat was held over three days at Breezy Ridge Retreat Centre, which is affiliated with a private cottage in Muskoka Ontario. To optimize engagement, no electronic devices were permitted during the sessions. The format of the retreat included structured and unstructured components. During the structured time, junior investigators presented their research protocols, which were followed by ample time for questions and personal reflection. All investigators were allocated 45 min to present their current progress and challenges to their peers and mentors in a format similar to that used at the CCCTG and CCCTBG meetings, ${ }^{7}$ although the timeline was relaxed to allow for more dialogue. The CCCTG and
CCCTBG meetings minimize presentation of data, study rationale, and background and focus more intently on optimizing methodology and overcoming practical challenges. An ensuing 45 min was allocated for focused peer-to-peer and group feedback. Feedback is always constructive and usually based on the previous experiences of the feedback provider in related research situations.

Interactive presentations by the mentors were interspersed among the participants' presentations. These talks were focused on key messages about developing a successful academic career. Unstructured time was allocated for impromptu exchanges, personal reflection, and outdoor leisure events that promoted social interactions among participants and between participants and mentors. New research collaborations and friendships were formed and existing relationships were strengthened during the retreat. Mentee feedback was sought informally throughout the retreat and formally during a closing session.

\section{Some of the key messages from the mentors}

Given all the challenges to be expected in an academic career, it is crucial to find and follow your passion.

Opportunities and expectations of young investigators abound when their careers begin; therefore, thoughtful prioritization of academic activities is key-"putting first things first". A tool to help realize this objective is to create a framework for organizing activities into those of higher vs lower importance and higher vs lower urgency. ${ }^{8}$ Prioritizing important activities, and urgent activities when necessary, and periodically reviewing this $2 \times 2$ table (known informally as an Eisenhower box) can help investigators become self-aware about how they spend their time. Revisiting this table and realigning activities if needed can explicitly help align (or realign) work with chief career goals.

Adequate training, sufficient designated time for research, and a clear and feasible research question are other key ingredients for academic success. It is wise to be mindful whether and when more training is needed (e.g., a new course, a refresher course, or formal degree) or a new collaborator with additional expertise is required to help advance a project.

Sometimes research hypotheses are flawed or goals are either unfeasible or perceived to be unfeasible. Therefore, young investigators need to be flexible, develop resilience, and handle setbacks. Obtaining peer-reviewed grants is difficult; nevertheless, investigators may learn considerably more from their failures than from their successes, and developing an entrepreneurial spirit is helpful. Constructively reading peer-reviews and incorporating feedback to improve grants are important skills to develop. 
Most research is a group activity; therefore, cultivating a spirit of inquiry among colleagues and deliberately building community around a project are energy-giving collaborative examples that foster shared values, encourage constructive criticism, and generate a team philosophy of working toward a common goal.

Clinical researchers are encouraged to collaborate with basic scientists to include a translational biology component in their research programs. The expertise and resources developed by the CCCTBG are available to young researchers to facilitate understanding the mechanisms of critical illness; discovery of diagnostic, prognostic, and therapeutic biomarkers; and development of new therapies.

Regardless whether young investigators hire their own staff colleagues or work with the existing staff of their mentor or group, an excellent environment focuses on staff career development and retention. The Research Coordinator group in Canada has a wealth of experience. The indispensable members of a research team can offer excellent advice about operationalizing protocols with "boots on the ground" insight. They also have valuable expertise in budgeting for local and central expenses as well as in research ethics and contracts.

\section{Some feedback from the mentees}

"I would recommend ANY early career researcher to actively seek out and apply to similarly structured retreats. The focused and condensed mentoring provided me with a huge amount of guidance and much needed feedback on my research program in a minimal time frame. The mentors and peer mentees were hugely supportive, respectful, and nonjudgemental, which both encouraged and challenged me to develop my skills and knowledge beyond their current state."

"The CCCTG YI Retreat provided a powerful academic growth experience for me. The relaxed approach at the retreat, the one-on-one time with the mentors, and built-in time for reflective practice provided the ideal environment to discuss topics such as efficient time organization, stress minimization practices, and effective communication tools. These research management skills are often overlooked or underemphasized during formal research training, but they are the foundation for any successful and rewarding academic career."

"The model of presentation /open feedback in an interprofessional setting was excellent for helping me to refine my research plan. There was enough time to go beyond surface-level questions and really get into critical questioning of the methods. After the retreat, I altered the
Table Senior researcher presentation topics

The importance of incorporating translational science into your research program

Being an effective mentor / mentee

Time management and important factors to consider when selecting research programs in which to participate

methodology in my CIHR application and was subsequently successful."

"This retreat was the first opportunity that I've had to speak with a female leader in critical care medicine about research plans, career goals, trajectory, and balance. The retreat was also great for introducing new female investigators. This is important because, at many institutions across the country, the number of men conducting research in critical care far outweighs the number of females."

The CCCTG Young Investigator's Retreat is intended to be held every two years and is intended to be supported by the Canadian Institutes of Health Research (Deborah Cook Foundation Grant and Community Development Grant of the CCCTG and CCCTBG).

\section{Conclusions}

Members of the Canadian critical care community understand that mentoring is shaped by the culture and climate of a group or institution; thus, organizational structure is needed to prioritize excellence in mentoring. The time required to mentor junior investigators may be under threat due to the increased administrative, clinical, research, and educational demands on medical faculty. ${ }^{9}$ Nevertheless, events such as the CCCTG Young Investigator Retreat are investments in researchers of the future. They provide a forum for project presentations, constructive feedback, and career advice in a collaborative, safe, and relaxed environment.

It is challenging to quantify the success of a program such as the CCCTG Young Investigator Retreat. Each attendee left with a self-discovered individualized work plan that included some modified methods and/or different project directions, priorities, and timelines. The CCCTG will be tracking the number of grants submitted and awarded as well as the participants' publications, career awards, academic appointments, and completed research projects and programs. More subjective outcomes, such as informal mentorships, new research collaborations, friendships, and the creation of a nurturing environment, are crucial for young researchers, albeit harder to quantify. 
Nevertheless, as Albert Einstein allegedly said: "Not everything that counts can be counted, and not everything that can be counted counts."

We look forward to seeing the participants of the CCCTG Young Investigator Retreat become excellent role models and eventually mentors for future investigators who will continue the legacy of critical care research in Canada (Table).

\section{La Retraite pour jeunes chercheurs du Groupe canadien de recherche en soins intensifs : mentorat et introspection}

Le Groupe canadien de recherche en soins intensifs (CCCTG) a été fondé en 1989 avec deux objectifs en tête : améliorer les soins aux patients en état critique grâce à la mise au point d'études à l'initiative des chercheurs, et proposer un forum national pour la formation continue en méthodologie. ${ }^{1}$ En 2004, un groupe de scientifiques en recherche clinique et fondamentale s'intéressant aux mécanismes de base des maladies critiques a créé le Groupe canadien de biologie translationnelle en soins intensifs (CCCTBG). Des 25 participants fondateurs en 1989, les deux groupes comptent aujourd'hui plus de 300 chercheurs. Le CCCTG et le CCCTBG se rencontrent et soutiennent actuellement plus de 40 programmes de recherche et ont plus de 100 publications révisées par les pairs à leur actif. Les deux ingrédients principaux de cette réussite sont la collégialité et le mentorat.

En recherche, un mentor peut jouer plusieurs rôles dans l'essor de la carrière d'un jeune chercheur : il peut être à la fois conseiller, défenseur, 'coach' ou superviseur, ou il peut épauler le mentoré en faisant preuve d'attention, d'engagement et d'esprit. Avec en tête la durabilité et la croissance de leur mission, le mandat explicite du CCCTG et du CCCTBG est de mentorer les nouveaux chercheurs aussi bien que les chercheurs plus aguerris de leurs rangs et de promouvoir leur réussite indépendante. Pour mener à bien ce mandat, diverses activités sont mises en œuvre: citons par exemple le modèle de «mentorat communautaire » de nos réunions trisannuelles, les liens explicites entre tout jeune chercheur qui présente ses travaux lors de ces réunions et un mentor du CCCTG, le fait d'encourager nos jeunes membres à participer aux comités nationaux, les bourses de recherche interdisciplinaire aux jeunes chercheurs, le Prix de mentorat Deborah J. Cook visant à honorer les personnes ayant apporté des contributions exceptionnelles à ce domaine, et la création de la Retraite pour jeunes chercheurs du CCCTG. Les hôtes de la première Retraite pour jeunes chercheurs du CCCTG étaient la Dre Deborah Cook et son conjoint Robert. Le format de cette retraite s'est largement inspiré des Ateliers au Trout Lake, fondés par feu Dr David Sackett, l'un des mentors les plus influents de Dre Cook et un pionnier de la médecine factuelle. ${ }^{2-6}$

\section{Financement et sélection des participants}

En 2014, le CCCTG a reçu une Bourse de développement communautaire des Instituts de recherche en santé du Canada (IRSC), dont une partie a été allouée au financement du mentorat. Les membres du CCCTG dans leurs cinq premières années de recherche ont été invités à postuler pour les premières Bourses de jeune chercheur en 2016. Le Comité de développement de carrière et de mentorat du CCCTG, composé de quatre membres, a passé en revue et évalué les candidatures (un résumé de l'étude proposée et une lettre du superviseur de recherche/mentor) en fonction de trois critères, soit l'impact potentiel de la retraite sur l'individu et son milieu de travail, la clarté et la faisabilité du résumé de l'étude proposée, et l'impact potentiel de la recherche proposée sur le pronostic des patients. Des participants provenant des quatre coins du Canada et de plusieurs disciplines professionnelles ont été sélectionnés par consensus, soit deux intensivistes en soins aux adultes, un neuro-intensiviste, un intensiviste pédiatrique, deux doctorants en pharmacie et un en sociologie. Cette approche multidisciplinaire se voulait le reflet de plusieurs programmes de recherche efficaces initiés par des chercheurs du CCCTG (un aspect fondamental de la Bourse de développement communautaire des IRSC). Les sept participants différaient de par leur sujet de recherche et leurs méthodes, qui comprenaient la recherche clinique et en services de santé ainsi que la recherche en préparation aux pandémies internationales. Trois chercheurs en soins intensifs bien établis (deux en soins adultes et un en pédiatrie) ont fait office de mentors. Ces mentors ont été sélectionnés en fonction de leurs antécédents d'excellence en qualité de chercheurs cliniques et de leur engagement reconnu dans l'enseignement et le mentorat.

Tableau Sujets des présentations des chercheurs mentors

De l'importance d'intégrer les sciences translationnelles dans votre programme de recherche

Comment être un bon mentor / mentoré

Gestion du temps et facteurs importants à prendre en compte lors de la sélection des programmes de recherche auxquels participer 


\section{Format de la retraite}

La retraite s'est tenue sur trois jours au Centre de retraite Breezy Ridge, un établissement affilié à un cottage privé à Muskoka, en Ontario. Afin de favoriser l'engagement des participants, aucun appareil électronique n'était permis pendant les sessions. Le format de la retraite comportait des certaines composantes structurées et d'autres non structurées. Pendant les périodes structurées, les jeunes chercheurs ont présenté leurs protocoles de recherche; s'ensuivait une longue période ouverte pour favoriser les questions et la réflexion personnelle. Chaque chercheur disposait de 45 minutes pour présenter ses progrès et les défis auxquels il faisait face à ses collègues et mentors, dans un format ressemblant celui utilisé lors des réunions du CCCTG et du CCCTBG, ${ }^{7}$ bien que le programme fut plus flexible afin d'encourager un dialogue plus approfondi. Les réunions du CCCTG et du CCCTBG tendent à minimiser le temps imparti à la présentation de données, à la justification de l'étude et au contexte afin de se concentrer davantage sur l'optimisation de la méthodologie et la résolution des difficultés pratiques. Les 45 minutes subséquentes étaient dédiées aux rétroactions ciblées entre pairs et en groupe. Les rétroactions étaient toujours constructives et se fondaient en général sur les expériences passées de la personne fournissant la rétroaction dans des situations de recherche similaires.

Des présentations interactives des mentors étaient disséminées entre les présentations des participants. Ces sessions se sont concentrées sur des messages clés visant à promouvoir le développement d'une carrière universitaire fructueuse. Du temps libre non structuré était prévu afin d'encourager les échanges impromptus, la réflexion personnelle et des activités de plein air, lesquelles avaient pour objectif de favoriser les interactions sociales entre participants et entre participants et mentors. De nouvelles collaborations de recherche et amitiés se sont ainsi créées au cours de la retraite, et les relations existantes se sont solidifiées. La rétroaction des mentorés a été sollicitée de manière informelle tout au long de la retraite, puis de manière formelle au cours d'une séance de clôture.

\section{Quelques messages clés des mentors}

Étant donné tous les défis auxquels on peut s'attendre au cours d'une carrière universitaire, il est essentiel de découvrir et de poursuivre sa passion.

Les occasions et les attentes des jeunes chercheurs abondent en début de carrière; ainsi, une priorisation réfléchie des activités universitaires est cruciale : il faut « commencer par le commencement». Un outil utile pour atteindre cet objectif est de créer un cadre afin d'organiser les activités selon leur importance, élevée $v s$ moindre, et leur urgence, élevée vs basse. ${ }^{8}$ La priorisation des activités importantes, et des activités urgentes lorsque nécessaire, ainsi que la révision périodique du tableau ainsi créé (connu sous le nom de matrice d'Eisenhower), peut aider les chercheurs à prendre conscience de la façon dont ils 'dépensent' leur temps. La révision de cette matrice et l'adaptation, au besoin, des activités peuvent aider, de façon explicite, à aligner (ou réaligner) le travail d'un individu sur ses plus importants objectifs de carrière.

Une formation adéquate, un temps alloué suffisant pour la recherche et une question de recherche claire et réalisable sont d'autres ingrédients clés de la réussite universitaire. Il est judicieux de porter attention à savoir si et quand une formation supplémentaire est nécessaire (par ex., un nouveau cours, un cours de recyclage, ou un diplôme), ou si un nouveau collaborateur possédant une expertise supplémentaire est requis pour faire avancer un projet.

Les hypothèses de recherche sont parfois imparfaites ou les objectifs irréalisables ou perçus comme tels. C'est pourquoi les jeunes chercheurs se doivent d'être flexibles, de développer leur résilience et de savoir faire face à l'adversité. L'obtention de bourses provenant de comités révisés par les pairs est difficile; cependant, un chercheur pourrait apprendre bien davantage de ses échecs que de ses réussites, et le développement d'un esprit d'entrepreneuriat est utile. La lecture constructive des révisions des pairs et l'intégration des rétroactions afin d'améliorer les bourses sont des qualités importantes à développer.

La plupart des recherches sont des activités de groupe; ainsi, le fait de cultiver un esprit curieux parmi ses collègues et de faire le choix délibéré de bâtir une communauté autour d'un projet sont des exemples de collaboration fructueuse qui soutiennent le développement de valeurs partagées, encouragent la critique constructive et génèrent une philosophie d'équipe pour travailler vers un but commun.

Les chercheurs cliniques sont encouragés à collaborer avec des scientifiques fondamentaux afin d'inclure une composante de biologie translationnelle dans leurs programmes de recherche. L'expertise et les ressources mises au point par le CCCTBG sont à la disposition des jeunes chercheurs afin de les aider à comprendre les mécanismes des maladies graves, découvrir un diagnostic, un pronostic et des biomarqueurs thérapeutiques, et à mettre au point de nouveaux traitements.

Que les jeunes chercheurs engagent leurs propres collègues ou travaillent avec le personnel existant de leur mentor ou de leur groupe, un environnement d'excellence favorisera le développement de carrière et la rétention du personnel. Le groupe des Coordonnateurs de recherche au 
Canada possède une riche expérience dans ce domaine. Les membres indispensables d'une équipe de recherche peuvent offrir de très bons conseils quant à la concrétisation de protocoles grâce à leur perspective «les pieds sur Terre ». Ils possèdent également une expertise précieuse en matière de budgétisation des dépenses locales et centrales ainsi qu'en éthique de la recherche et en contrats.

\section{Quelques commentaires des mentorés}

« Je recommanderais à TOUT chercheur en début de carrière d'être proactif dans ses recherches et de postuler pour participer à des retraites de ce genre. Les sessions de mentorat ciblées et condensées m'ont donné une quantité incroyable de conseils et des rétroactions plus que nécessaires et bienvenus concernant mon programme de recherche et ce, dans un minimum de temps. Les mentors et autres mentorés ont tous fait preuve d'énormément de soutien, de respect et ce, sans jugement, ce qui m'a à la fois encouragé et mis au défi de pousser mes compétences et mes connaissances plus loin. »

« La Retraite pour jeunes chercheurs du CCCTG fut une expérience puissance de croissance didactique pour moi. L'approche détendue de la retraite, le temps individuel passé avec les mentors, et le temps alloué à la réflexion personnelle ont tous contribué à créer un environnement idéal pour discuter de thèmes tels qu'une organisation efficace du temps, les pratiques de minimisation du stress, et les outils de communication efficaces. Ces compétences de gestion de la recherche sont souvent ignorées ou minimisées pendant la formation en recherche formelle, mais elles constituent le fondement de toute carrière universitaire réussie et gratifiante. »

« Le modèle de présentation / rétroaction ouverte dans un contexte interprofessionnel fut excellent pour m'aider à raffiner mon plan de recherche. Un temps suffisant était imparti pour aller au-delà des questions de surface et véritablement approfondir le questionnement des méthodes. Après la retraite, j'ai modifié la méthodologie de ma candidature pour un financement des IRSC et j'ai réussi à l'obtenir. »

«Cette retraite a été la première occasion pour moi de parler à une femme chef de file en soins intensifs concernant mes plans de recherche, mes objectifs de carrière, ma trajectoire, et la recherche d'un équilibre. La retraite était également excellente car elle a présenté de nouvelles femmes chercheures. Ceci est important parce que, dans plusieurs établissements partout au pays, le nombre d'hommes faisant de la recherche en soins critiques dépasse de loin le nombre de femmes. »
La Retraite pour jeunes chercheurs du CCCTG devrait se tenir tous les deux ans et être financée par les Instituts de recherche en santé du Canada (Bourse de la Fondation Deborah Cook et Bourse de développement communautaire du CCCTG et du CCCTBG).

\section{Conclusion}

Les membres de la communauté canadienne des soins intensifs sont conscients que le mentorat est façonné par la culture et le climat d'un groupe ou d'une institution; ainsi, une structure organisationnelle est nécessaire pour prioriser l'excellence dans le mentorat. Le temps nécessaire au mentorat de jeunes chercheurs pourrait être mis en péril en raison de l'augmentation des exigences administratives, cliniques, de recherche et éducationnelles imposées au personnel médical. ${ }^{9}$ Toutefois, les événements tels que la Retraite pour jeunes chercheurs du CCCTG sont un investissement dans les chercheurs du futur. Ils constituent un forum visant à encourager les présentations de projets, les rétroactions constructives et des conseils de carrière dans un environnement collaboratif, sécuritaire et détendu.

Il est difficile de quantifier le succès d'un programme tel que la Retraite pour jeune chercheur du CCCTG. Chaque participant est reparti avec un plan de travail personnalisé qu'il a mis au point lui-même et qui comprenait des méthodes modifiées et/ou différentes orientations de projet, priorités ou calendriers. Le CCCTG suivra le nombre de bourses soumises et accordées ainsi que les publications, prix de carrière, nominations universitaires et projets et programmes de recherche complétés des participants. Des résultats plus subjectifs, tels que des mentorats informels, de nouvelles collaborations de recherche, des amitiés et la création d'un environnement propice sont cruciaux pour les jeunes chercheurs, même si ces éléments sont plus difficiles à quantifier. Cependant, comme Albert Einstein l'a supposément dit: "Tout ce qui compte ne peut pas nécessairement être compté, et tout ce qui peut être compté ne compte pas nécessairement. »

Nous nous réjouissons de voir les participants à la Retraite pour jeunes chercheurs du CCCTG devenir d'excellents modèles à émuler et, à leur tour, des mentors pour les chercheurs futurs qui poursuivront l'héritage de la recherche en soins intensifs au Canada (Tableau).

Acknowledgements We appreciate the organizational help of Nicolay Ferrari, Elaine Gilfoyle, Dominique Piquette, Paul Hébert, and Robert Sheppard. This retreat was partially supported by the Canadian Institutes of Health Research (Deborah Cook Foundation Grant and Community Development Grant of the CCCTG and CCCTBG). 
Conflicts of interest None declared.

Editorial Responsibility This submission was handled by Dr. Gregory L. Bryson, Deputy Editor-in-Chief, Canadian Journal of Anesthesia.

Remerciements Nous tenons à souligner l'aide organisationnelle de Nicolay Ferrari, Elaine Gilfoyle, Dominique Piquette, Paul Hébert et Robert Sheppard. La retraite a été financée en partie par les Instituts de recherche en santé du Canada (Bourse de la Fondation Deborah Cook et Bourse de développement communautaire du CCCTG et du CCCTBG).

\section{Conflit d'intérêt Aucun.}

Responsabilité éditoriale Cet article a été traité par Dr Gregory L. Bryson, rédacteur en chef adjoint, Journal canadien d'anesthésie.

\section{References}

1. Cook DJ, Todd TR. The Canadian Critical Care Trials Group: a collaborative educational organization for the advancement of adult clinical ICU research. Intensive Care World 1997; 14: 68-70.
2. Chalmers I, Oxman AD. Farewell and thanks to Dave Sackett. Cochrane's first pilot. J Clin Epidemiol 2016; 73: 58-60.

3. Straus $S E$, Haynes RB. David Sackett's legacy includes evidencebased mentorship. J Clin Epidemiol 2016; 73: 61-3.

4. Straus SE, Sackett DL. Mentorship in Academic Medicine. UK: John Wiley \& Sons, Ltd; 2013: i-xiv.

5. Evidence-Based Medicine Working Group. Evidence-based medicine. A new approach to teaching the practice of medicine. JAMA 1992; 268: 2420-5.

6. Guyatt GH. Evidence-based medicine. Ann Intern Med 1991; 114 A-16.

7. Marshall JC. Cook DJ; Canadian Critical Care Trials Group. Investigator-led clinical research consortia: the Canadian Critical Care Trials Group. Crit Care Med 2009; 37: S165-72.

8. Covey SR. The 7 Habits of Highly Effective People: Powerful Lessons in Personal Change, $25^{\text {th }}$ Anniversary Edition. Village Books/ Tsai Fong Books; 2013.

9. Sambunjak D, Straus SE, Marusic A. A systematic review of qualitative research on the meaning and characteristics of mentoring in academic medicine. J Gen Internal Med 2010; 25: 72-8. 